\title{
Editorial
}

\section{Non-Selective Laryngeal Reinnervation for Unilateral Vocal Fold Paralysis}

\author{
Marina MB
}

Department of Otorhinolaryngology, Faculty of Medicine, Universiti Kebangsaan Malaysia Medical Centre, Jalan Yaacob Latif, Bandar Tun Razak, 56000 Cheras, Kuala Lumpur, Malaysia.

\section{Introduction}

Unilateral vocal fold paralysis (UVFP) is a nightmare for professional voice users or people with high voice demand such as singers, lawyers, teachers, training consultants and etc. The UVFP occurs due to physical or physiological injury to the vagus nerve or, more commonly, its branch, the recurrent laryngeal nerve (RLN). Common aetiologies of UVFPare iatrogenic, neoplastic and idiopathic. However, the commonest cause is iatrogenic including thyroid surgery, carotid endarterectomy, anterior approaches to the cervical spine, and heart or great vessel surgery (1). Risk of permanent vocal fold palsy following thyroidectomy has been estimated at 1-2\% (2) but this may be a considerable underestimate due to problems of followup and reporting.

In UVFP, the affected vocal fold is flaccid and incapable of closing the glottis during speech. The vocal folds cannot make a firm contact to produce a strong voice. The voice is frequently breathy and easily fatigued (3). This problem may also lead to ineffective protective mechanisms by the larynx during swallowing causing aspiration that subsequently may lead to aspiration pneumonia. Symptomatic patients with UVFP suffer reduced general health and quality of life due to social and emotional disturbance as a consequence of the voice disorder. Therefore, rehabilitation of the paralysed larynx is important to patients.

Rehabilitation of the paralysed larynx includes surgical treatments that the aim is to achieve firm contact between two vocal folds during voice production. The proposed treatment options are- injection laryngoplasty, Isshiki type I thyroplasty, arytenoid adduction and laryngeal reinnervation (4-7).

\section{Laryngeal Reinnervation}

A novel surgical approach for vocal fold paralysis is laryngeal reinnervation in which a donor nerve that is a foreign adjacent nerve, connected to a denervated muscle to re-establish working motor endplates and produce muscular contraction (8). The adjacent nerves for reinnervation which were reported in the literature are the ansa cervicalis, hypoglossal, superior laryngeal and phrenic. There are two main types of laryngeal reinnervation: 1) non-selective; and 2) selective (9). The selective reinnervation is mainly for bilateral vocal fold paralysis when airway is an issue and the aim is to re-establish mobility of the vocal fold to improve the glottis opening (10). On the other hand, non-selective reinnervation is for UVFP when voice is an issue and the aim is to re-establish tone and bulk of the vocal fold muscles (11).

Non-selective reinnervation techniques described in the literature include nerve-to-nerve anastomosis (4), nerve-muscle pedicle (12) and nerve implant (13). The nerve-to-nerve anastomosis involves connecting a donor nerve to the viable distal end of the RLN. The nerve-muscle pedicle technique uses a donor nerve attached to a small block of 'donor' muscle surrounding its terminal portion. The nerve implant technique is the modification of Tucker's technique whereby the nerve ending is implanted directly into the laryngeal muscle. The most common technique reported is nerve-to-nerve anastomosis employing ansacervicalis as the donor nerve as described by Crumley (ansa-RLN) (14). Wang et al. (15) had 
improved the technique by using the main branch of the ansa cervicalis that has more axons to regenerate to the injured RLN instead of ansa hypoglossi to sternohyoid, and they described details of the surgery for a successful reinnervation.

Nerve 'anastomosis' is actually not an accurate term as it is supposed to be used in describing connection of two luminal structures such as bowel, blood vessels, bile ducts and others. However, nerve anastomosis is a common misuse in the literature related to reinnervation surgery.

\section{Voice Outcomes}

A systematic review of studies on reinnervation in UVFP patients was done to investigate the effectiveness in improving the voice (14). The authors extracted 14 papers out of 686 papers retrieved from literature databases that met the inclusion and exclusion criteria. This review revealed that reinnervation is effective in improving voice to a varying degree. In another study, significant long-term voice improvement was documented in UVFP patients when the results were analysed between pre- and postoperative. The successful results were supported by the physiological evidence on laryngeal electromyography (16).

Wang et al. had included the largest number of patients. In this study, the success rate of ansa-RLN technique was found to be high with only $2 \%$ of failure to achieve satisfactory vocal function. The rate of major complications involving airway compromise was low ( 2 of 237 patients). None of these patients developed paradoxical vocal fold mobility (15).

\section{Advantages against Other Surgical Techniques}

Non-selective laryngeal reinnervation has the potential of restoring normal or near normal voice in UVFP patients. There are several advantages of laryngeal reinnervation over other surgical techniques. Specifically, it avoids vocal fold atrophy and provides a long-term solution by maintaining the vocal fold mass, bulk, tone and pliability for normal mucosal waves. The possibility of other forms of laryngoplasty is preserved should voice outcomes not reach desired levels.

Journal of Surgical Academia 2016;6(2):1-3
Quality of voice is determined by mass, elasticity, resistance and symmetry of the vocal folds, all of which are affected in UVFP. Denervation of the PCA and interarytenoidmuscle causes anterior subluxation of the arytenoid and vocal process causing asymmetry of the vocal folds. Reinnervating the paralysed laryngeal muscles in UVFP employs the principle of gaining favourable synkinesis (16) that will improve the resting tone and muscle bulk as well as restore the arytenoid to an upright position thus reducing the glottal incompetence (17). Firm contact of the two folds allows synchronous oscillation of the mucosa, reducing aperiodicity and perturbation of voice.

This procedure avoids manipulation of the vocal fold therefore the mucosa remains supple and pliable. It precludes the need of implant adjustment to fine-tune the voice and this makes it suitable even in children. Furthermore, it preserves the possibility of other medialisation technique in future if it fails.

Non-selective laryngeal reinnervation is performed not only in delayed setting when the UVFP is diagnosed post-operatively but as the same setting as well when accidental transection of RLN occurred or when the vagus nerve was intentionally removed in vagal schwannoma or paraganglioma.

Disadvantages of this technique are the requirement of a general anaesthetic, voice improvement is not immediate, requirement of an intact nerve donor and recipient and a suggestion of increased probability of delayed or failed reinnervation in older patients (18). Although the effect of reinnervation is delayed up to 4 months, the problems of aspiration and hoarseness may be assisted temporarily by bulking up the vocal fold with absorbable injectable substances- injection laryngoplasty. Concomitant injection laryngoplasty using absorbable material may give immediate improvement, strengthening the voice during this waiting period (19).

\section{Conclusion}

Non-selective laryngeal reinnervation is a surgical option for patients with UVFP that may prevent denervation atrophy of the paralysed vocal fold muscles and bring back normal or near-normal voice 
as well as alleviate symptoms of aspiration. It is pertinent to preserve the ansa cervicalis nerve during neck surgery when RLN is at risk as it is the most frequent donor nerve been used for the reinnervation procedure.

\section{References}

1. Rosenthal LH, Benninger MS, Deeb RH. Vocal fold immobility: a longitudinal analysis of etiology over 20 years. Laryngoscope 2007; 117(1): 1864-70.

2. Jeannon JP, Orabi AA, Bruch GA, Abdalsalam HA, Simo R. Diagnosis of recurrent laryngeal nerve palsy after thyroidectomy: a systematic review. Int J ClinPract 2009; 63(4): 624-9.

3. Misono S, Merati AL. Evidence-based practice: evaluation and management of unilateral vocal fold paralysis. Otolaryngol Clin North Am 2012; 45(5): 1083-108.

4. Crumley RL, Izdebski K. Voice quality following laryngeal reinnervation by ansa hypoglossi transfer. Laryngoscope 1986; 96(6): 611-6.

5. Isshiki N, Okamura H, Ishikawa T. Thyroplasty type I (lateral compression) for dysphonia due to vocal cord paralysis or atrophy. Acta Otolaryngologica 1975; 80(5-6): 465-73.

6. Isshiki N, Tanabe M, Sawada M. Arytenoid adduction for unilateral vocal cord paralysis. Arch Otolaryngol 1978; 104(10): 555-8.

7. McCulloch TM, Hoffman HT. Medialization laryngoplasty with expanded polytetrafluoroethylene. Surgical technique and preliminary results. Ann Otol Rhinol Laryngol 1998; 107(5 Pt 1): 427-32.

8. Crumley RL. Experiments in laryngeal reinnervation. Laryngoscope 1982; 92(9 Pt 2 Suppl 30): 1-27.

9. Marina MB, Marie JP, Birchall MA. Laryngeal reinnervation for bilateral vocal fold paralysis. Curr Opin Otolaryngol Head Neck Surg 2011; 19(6): 434-8.
10. Marie JP, Dehesdin D, Ducastelle T, Senant J. Selective reinnervation of the abductor and adductor muscles of the canine larynx after recurrent nerve paralysis. Ann Otol Rhinol Laryngol 1989; 98(7 Pt 1): 530-6.

11. Crumley RL. Update: ansa cervicalis to recurrent laryngeal nerve anastomosis for unilateral laryngeal paralysis. Laryngoscope 1991; 101(4 Pt 1): 384-7; discussion 388.

12. Tucker HM. Human laryngeal reinnervation. Laryngoscope 1976; 86(6): 769-79.

13. Su WF, Hsu YD, Chen HC, Sheng H. Laryngeal reinnervation by ansa cervicalis nerve implantation for unilateral vocal cord paralysis in humans. J Am CollSurg 2007; 204(1): 64-72.

14. Aynehchi BB, McCoul ED, Sundaram K. Systematic review of laryngeal reinnervation techniques. Otolaryngol Head Neck Surg 2010; 143(6): 749-59.

15. Wang W, Chen D, Chen S, et al. Laryngeal reinnervation using ansa cervicalis for thyroid surgery-related unilateral vocal fold paralysis: a long-term outcome analysis of 237 cases. PLoS ONE 2011; 6(4): e19128.

16. Crumley RL. Laryngeal synkinesis revisited. Ann Otol Rhinol Laryngol 2000; 109(4): 365-71.

17. Maronian N, Waugh P, Robinson L, Hillel A. Electromyographic findings in recurrent laryngeal nerve reinnervation. Ann Otol Rhinol Laryngol 2003; 112(4): 314-23.

18. Lee WT, Milstein C, Hicks D, Akst LM, Esclamado RM. Results of ansa to recurrent laryngeal nerve reinnervation. Otolaryngol Head Neck Surg 2007; 136(3): 450-4.

19. Lee WT, Milstein C, Hicks D, Akst LM, Esclamado RM. Results of ansa to recurrent laryngeal nerve reinnervation. Otolaryngol Head Neck Surg 2007; 136(3): 450-4. 